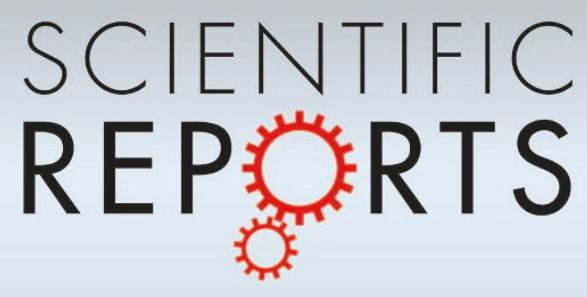

OPEN

SUBJECT AREAS:

NONLINEAR

PHENOMENA

MECHANICAL ENGINEERING

Received

11 December 2013

Accepted

3 March 2014

Published

17 March 2014

Correspondence and requests for materials should be addressed to

T.K. (†omaszka@p. lodz.pl)

\title{
Synchronization extends the life time of the desired behavior of globally coupled
}

\section{systems}

\author{
Marcin Kapitaniak',2, Mateusz Lazarek', Michal Nielaczny' ', Krzysztof Czolczynski', \\ Przemyslaw Perlikowski' \& Tomasz Kapitaniak'
}

'Division of Dynamics, Technical University of Lodz, Stefanowskiego 1/15, 90-924 Lodz, Poland, ${ }^{2}$ Centre for Applied Dynamics
Research, School of Engineering, University of Aberdeen, AB24 3UE, Aberdeen, Scotland.

Synchronization occurs widely in natural and technological world, but it has not been widely used to extend the life time of the desirable behavior of the coupled systems. Here we consider the globally coupled system consisting of $\boldsymbol{n}$ units and show that the initial synchronous state extends the lifetime of desired behavior of the coupled system in the case when the excitation of one or few units is suddenly (breakdown of energy supply) or gradually (as the effect of aging and fatigue) switched off. We give evidence that for the properly chosen coupling the energy transfer from the excited units allows unexcited units to operate in the desired manner. As proof of concept, we examine the system of coupled externally excited rotating pendula. After the partial excitation switch off the initial complete synchronization of all pendula is replaced by phase synchronization with a constant phase shift between the clusters of excited and unexcited pendula. Our results show that the described extension of the system's life time occurs for the wide range of coupling parameters and is robust to the external perturbations.

T he concept of synchronized behavior in coupled systems is pervasive in both nature ${ }^{1}$ and physical systems ${ }^{2}$. Examples of synchronization include rhythmic blinking of fireflies ${ }^{3}$, rhythmic hands clapping after a musical performance ${ }^{4}$, crowd synchrony on the oscillating bridge ${ }^{5,6}$, and the spin-orbit resonance of the planet Mercury $^{7}$. In physical systems, synchronization has been studied for more than three centuries, starting with Huygens' experiment with two coupled pendulum clocks ${ }^{8,9}$ and leading to modern-day experiments of coupled nano-oscillators ${ }^{10-12}$ and chaos based communication ${ }^{13,14}$. These examples illustrate fundamental elements of synchronization: the individual units display periodic motion, they display adjustable phase and frequency, and they couple ${ }^{15}$.

Consider the system of $n$ globally coupled excited identical units as shown in Figure 1(a,b) (example for $n=6$ ). For the appropriately chosen coupling such a system can reach the state of complete synchronization ${ }^{2,15}$ (all the units behave identically). However, in such a system it is possible that some units suddenly (breakdown of energy supply) or gradually (as the effect of aging and fatigue) loss their excitation. Here, we show that in such case the synchronization can extend the life time of the desirable behavior of the coupled system. As proof of concept, we examine the mechanical implementation of globally coupled system, i.e., the system of $n$ rotating pendula mounted to the beam which can move in horizontal direction as shown in Figure 1(a,b) (example for 6 pendula) and assume that the rotation of all pendula is a desired behavior of coupled system. Initially all pendula are excited and reach complete synchronization as shown in Figure 1(a), them the excitation of some pendula is swiched off. We show that all pendula can still rotate but the unexcited pendula (two green pendula in Figure 1(b)) are phase shifted with the excited pendula (three red pendula in Figure 1(b)).

The selection of this example has been motivated by the studies of Blekhman ${ }^{16}$ who considers the case of two identical (or nearly identical) unbalanced rotors mounted on the oscillating base. It has been shown the oscillations of the base can maintain the rotation of one rotor when its excitation is switched off (effect of the oscillatory maintenance of rotation which is also used in the well-known game 'hoola-hoop'). Contrary to these studies we are not assuming weak coupling between pendula and not using small parameter method which for example do not allow identification of co-existing synchronous states ${ }^{17,18}$. Our considerations of two synchronized pendula with significantly different masses allow generalization of our results to the case on $n$ pendula.

In our studies we consider the system shown in Figure 2(a). It consists of a rigid beam of mass $m_{\mathrm{B}}$ on which $n$ rotating pendula are mounted. In Figure $3(\mathrm{a}, \mathrm{b})$ the beam is connected to a stationary base by the spring (or 

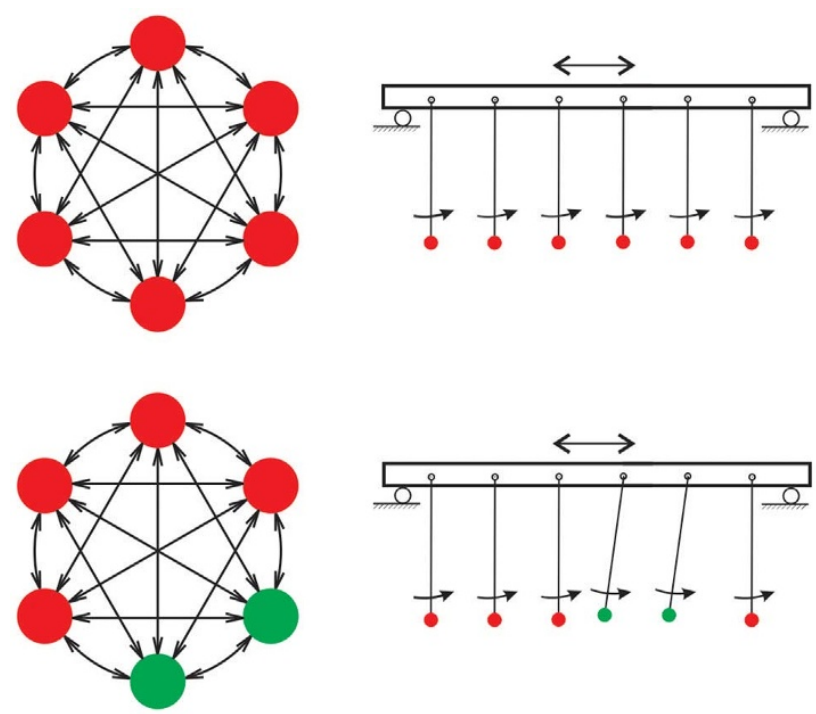

Figure 1 System of 6 globally coupled units and its mechanical implementation as a system of 6 externally forced pendula mounted to the beam which can move horizontally, (a) all units (pendula) are externally excited (red color) and synchronized, (b) two units (green color are not excited - the cluster of four excited pendula is phase synchronized with the cluster of two unexcited pendula. springs) with stiffness coefficient $k_{x}$ and a damper (or dampers) with a damping coefficient $c_{x}$. Due to the existence of the forces of inertia, which act on each pendulum pivot, the beam can move in horizontal directions (this motion is described by coordinate $x$ ). The masses of the pendula are indicated as $m_{\mathrm{i}} ; l_{\mathrm{i}}$ are the lengths of the pendula. The rotation of the $i$-th pendula is described by $\varphi_{i}$. The rotations of the pendula are damped by linear dampers with damping coefficient $c_{\varphi i}$. Each pendulum is driven by the drive torque inversely proportional to its velocity: $p_{0 i}-\dot{\varphi}_{i} p_{1 i}$. If any other external forces do not act on the pendulum, then under the action of such a torque it rotates with constant angular velocity $\dot{\varphi}_{i}$. As the system is in a gravitational field ( $g=9.81\left[\mathrm{~m} / \mathrm{s}^{2}\right]$ - acceleration of gravity), the weight of the pendulum causes the unevenness of its rotation: the pendulum slows down, when the center of mass rises up and accelerates when the center of mass falls down. The effect of gravity is important in the case of slow rotations of the pendula ${ }^{16-18}$. To explain how the synchronization can be achieved in the systems of Figure 2(a) first consider the case of identical pendula and nonmovable beam. In this case all pendula have the same period of rotations (the pendula have the same masses and lengths). The rotations of the pendula are initiated by non-zero initial conditions and the pendula's evolutions tend to the limit cycles. The pendula are not coupled and the phase angles between their displacements have fixed values, depending on initial conditions. Any perturbation of the pendula results in the changes of these angles. When the beam can move, its oscillations excited by the forces with which pendula act on it, cause the changes of the phase shifts between the pendula's displacements and differentiate the angular velocity of their rotations. When after the transient time, all pendula have the same angular velocity of rotation and there are constant phase shifts between the pendula's displacements, we

(a)

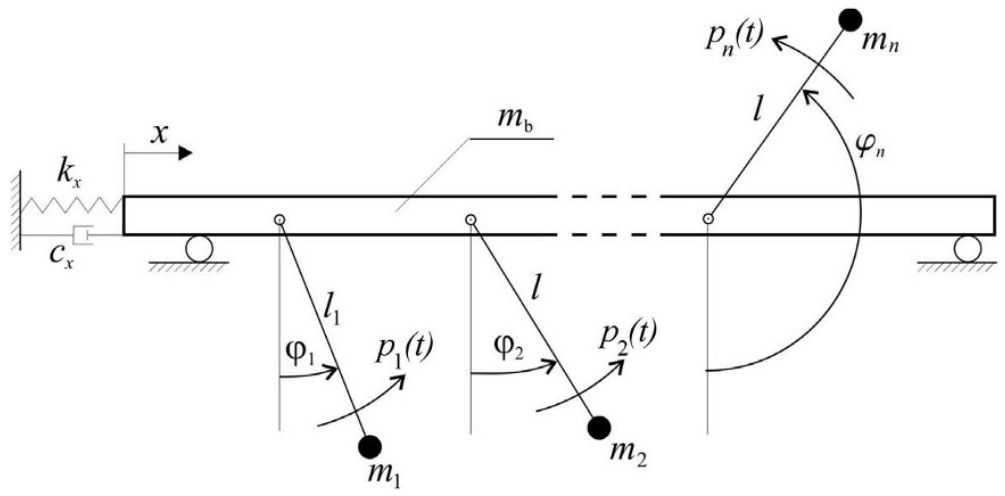

(b)

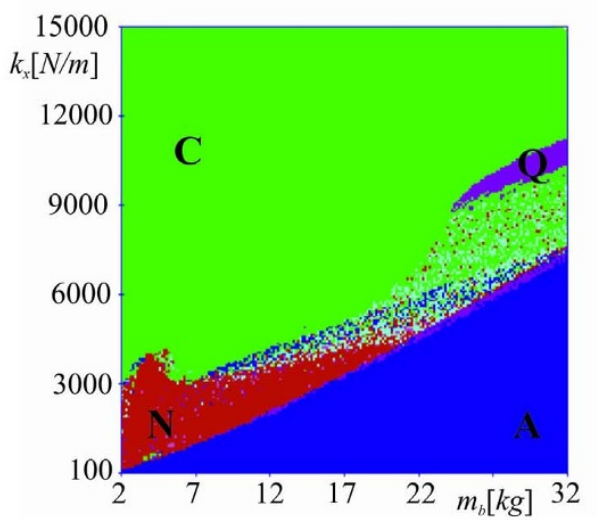

(c)

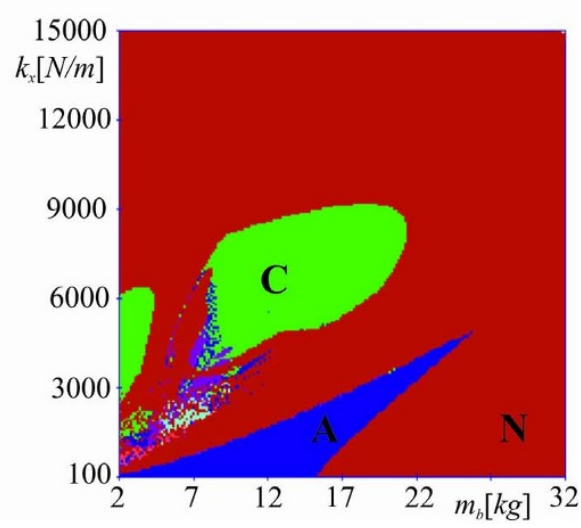

Figure $2 \mid$ (a) $n$ externally forced pendula mounted to the beam which can move horizontally; (b,c) The regions of the parameters $k_{\mathrm{x}}-m_{\mathrm{b}}$ space of complete C (green), antiphase A (navy blue), quasiperiodic Q (violet) synchronizations for the system of two pendula with different masses rotating in the same direction; $\mathrm{N}$ indicated the region in which pendulum 2 stops, $l=0.25[\mathrm{~m}], c_{\varphi}=0.03[\mathrm{Nsm}], p_{01}=p_{02}=5.0[\mathrm{Nm}]$, $p_{11}=p_{12}=0.2[\mathrm{Nms}],(\mathrm{b}) m_{1}=1.9[\mathrm{~kg}]$ and $m_{2}=0.1[\mathrm{~kg}]$, after the initial time equal to $50 \mathrm{~N}$, excitation of pendulum 2 is switched off, $(\mathrm{c}) m_{1}=0.9[\mathrm{~kg}]$ and $m_{2}=1.1[\mathrm{~kg}]$, after the initial time equal to $50 \mathrm{~N}$, excitation of pendulum 2 is switched off. 

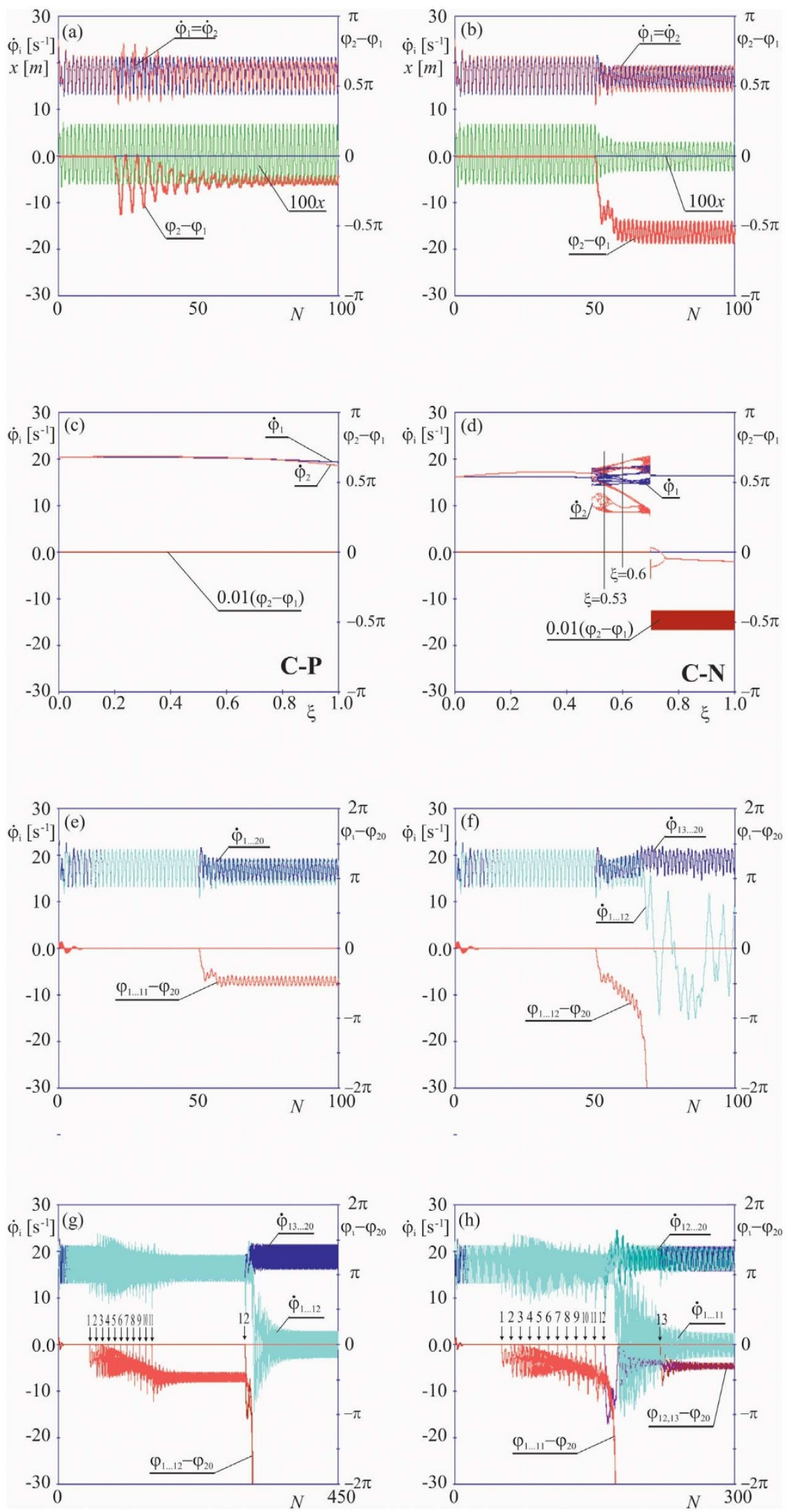

Figure $3 \mid$ (a,b,e,f,g,h) Time series showing the transient behavior of pendula's angular velocities $\dot{\varphi}_{i}$, the difference of pendula's displacement $\varphi_{j}-\varphi_{i}$ $(i \neq j)$ and beam's displacement $x$ (magnified 100 times) in the case when the excitations of a number of pendula are switched off: $p_{01}=5.0, p_{11}=0.2$, $p_{02}=5.0, p_{12}=0.2, p_{21}=p_{22}=0, k_{\mathrm{x}}=7000.0[\mathrm{~N} / \mathrm{m}], m_{\mathrm{b}}=12.0[\mathrm{~kg}],(\mathrm{a}) n=2, m_{1}=1.9[\mathrm{~kg}], m_{2}=0.1[\mathrm{~kg}]$, the excitation of pendulum $2 \mathrm{switched}$ off at $\left.N=20,(\mathrm{~b}) m_{1}=0.85[\mathrm{~kg}], m_{2}=1.15[\mathrm{~kg}]\right)$ the excitation of pendulum 2 switched off at $50 \mathrm{~N}$. (c,d) Bifurcation diagrams of pendula's angular velocities $\dot{\varphi}_{1}, \dot{\varphi}_{2}$ and the difference of pendula's displacement $\varphi_{2}-\varphi_{1}$ versus parameter $\xi$, excitation of pendulum 2 gradually decays to zero, i.e., $(1-\xi)\left(p_{02}-\dot{\varphi}_{2} p_{12}\right), n=2, m_{1}=1.9[\mathrm{~kg}], m_{2}=0.1[\mathrm{~kg}](\mathrm{c}) k_{\mathrm{x}}=7000.0[\mathrm{~N} / \mathrm{m}], m_{\mathrm{b}}=12.0[\mathrm{~kg}],(\mathrm{d}) k_{\mathrm{x}}=3700.0[\mathrm{~N} / \mathrm{m}], m_{\mathrm{b}}=18.0[\mathrm{~kg}],(\mathrm{e}-\mathrm{h}) n=20$, identical pendula with mass $m_{1-20}=0.1,(\mathrm{e}, \mathrm{f})$ at $N=50$, excitation of $p$ pendula is simultaneously switched off, $(\mathrm{e}) p=11,(\mathrm{f}) p=12,(\mathrm{~g}, \mathrm{~h}) n=20$ identical pendula, $m_{1-20}=0.1[\mathrm{~kg}]$, at the moments indicated by the arrows the excitations of one pendulum is switched off, (g) excitation of 11 pendula is switched off at $N=50,60, \ldots 150$, excitation of the 12-th pendulum is switched off at $N=300$, (h) excitation of 12 pendula are switched off for $N=50,60, \ldots 150,160$, excitation of the 13-th pendulum is switched off for $N=220$. 
(a)

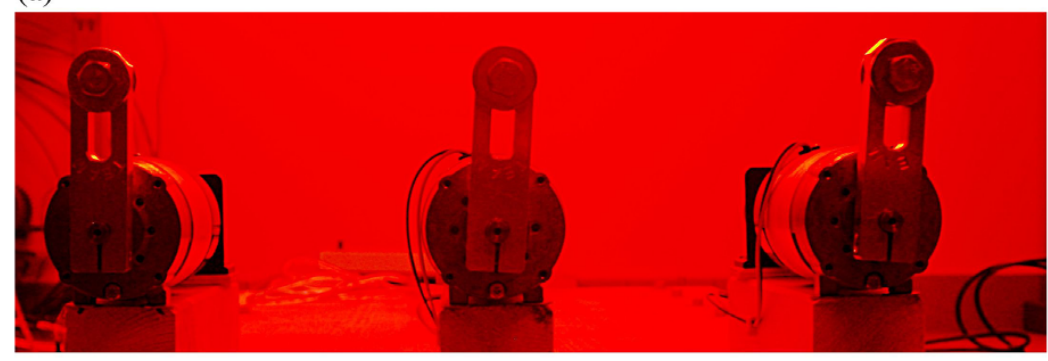

(b)

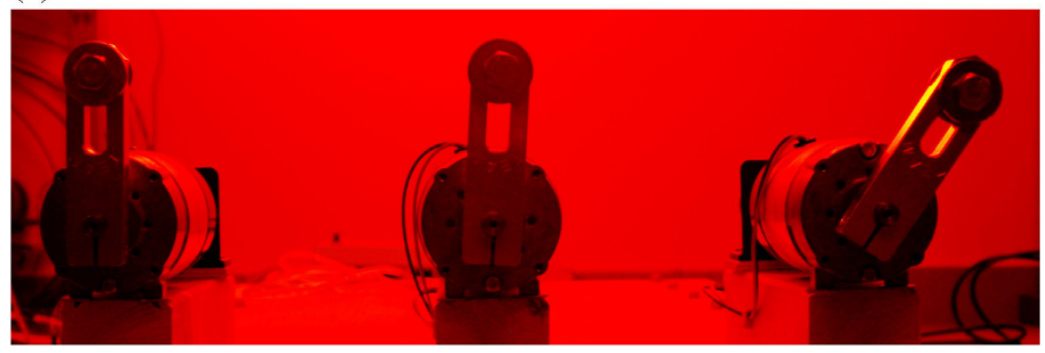

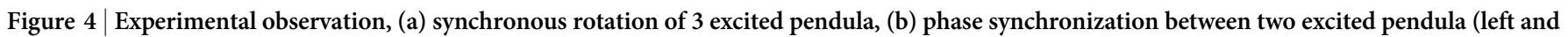
middle) and unexcited pendulum (right).

can say that the pendula achieve synchronization. The state of synchronization is achieved when the motion of the system is periodic and there are constant phase shifts between the pendula displacements ${ }^{16-18}$. The values of the phase shifts characterize the synchronous configuration and are independent of the initial conditions.

First let us consider the case of two pendula with different masses $\left(m_{1} \neq m_{2}\right)$. Figure 2(b,c) shows the dependence of the synchronous configuration on the parameters $k_{\mathrm{x}}$ and $m_{\mathrm{b}}$. After the initial time $N=$ 50 during which the pendula reach complete (phase shift between pendula is equal to zero), antiphase (phase shift between pendula is equal to $\pi$ ) or quasiperiodic (pendula perform identical quasiperiodic rotation) synchronizations the excitation of pendulum 2 is switched off. Figure 2(b) illustrates the case of $m_{1}=1.9[\mathrm{~kg}]$ and $m_{2}=0.1[\mathrm{~kg}]$. In regions indicated in green, navy blue and violet colors the pendula are respectively in the state of complete $\mathrm{C}$, antiphase A, quasiperiodic $Q$ synchronizations when the excitation of pendulum 2 is switched off. In region $\mathrm{N}$ pendulum 2 stops. The synchronization and the rotation of both pendula are preserved but the complete synchronization is replaced by phase synchronization (phase shift not equal to zero or $\pi$ ). For the wide range of $k_{x}$ and $m_{b}$ parameters the desired behavior of the coupled system is preserved. Contrary to this for $m_{1}=0.9[\mathrm{~kg}]$ and $m_{2}=1.1[\mathrm{~kg}]$ the set parameters for which both pendula rotate is very small as shown in Figure 2(c). Notice that in this case the excitation of the heavier pendulum has been switched off.

Figure 3(a,b) shows time series of the transient behavior of pendula's angular velocities $\dot{\varphi}_{1}, \dot{\varphi}_{2}$, the difference of pendula's displacement $\varphi_{2}-\varphi_{1}$ and beam's displacement $x$ (magnified 100 times) in the case when the excitation of pendulum 2 is switched off $\left(m_{1}=\right.$ $1.9[\mathrm{~kg}], m_{2}=0.1[\mathrm{k}]$ and $\left.m_{\mathrm{b}}=12.0[\mathrm{~kg}], k_{\mathrm{x}}=7000[\mathrm{~N} / \mathrm{m}]\right)$. Both pendula are in the state of complete synchronization when at time $20 \mathrm{~N}$ the excitation of pendulum 2 is switched off. After the transient time the system reaches the state of phase synchronization with nonzero phase shift between pendula. Both pendula rotate with the same angular velocity (smaller that before the switch off). Figure 3(b) shows the case for $m_{1}=0.85[\mathrm{~kg}]$ and $m_{2}=1.15[\mathrm{~kg}]$. At time $20 \mathrm{~N}$ the excitation of pendulum 2 is switched off. Synchronization and rotation of both pendula are preserved but the phase shift $\varphi_{2}-\varphi_{1}$ increases to the value larger than $\pi / 2$. Further increase of the difference of pendula's masses (down to $m_{1}=0.83[\mathrm{~kg}]$ and $m_{2}=$ $1.17[\mathrm{~kg}]$ ) leads to the loss of synchronization and pendulum 2 stops.

Now let us consider the case when the excitation of pendulum 2 gradually decays to zero. The excitation decay can be described as $(1-\xi)\left(p_{02}-\dot{\varphi}_{2} p_{12}\right)$, where $\xi(\xi \in[0,1])$ is a control parameter. The bifurcation diagrams shown in Figure 3(c,d) present the values of the pendula's angular velocities $\dot{\varphi}_{1}, \dot{\varphi}_{2}$ (at the moments when pendulum 1 moves through the lower equilibrium position) and the difference of pendula's displacement $\varphi_{2}-\varphi_{1}$ versus parameter $\xi$. In the case of Figure $3(\mathrm{c})\left(k_{\mathrm{x}}=7000.0[\mathrm{~N} / \mathrm{m}], m_{\mathrm{b}}=12.0[\mathrm{~kg}]\right.$, both pendula are initially in the state of complete synchronization) synchronization is preserved up to the value $\xi=0.85$. The phase shift between pendula is visible for larger values of $\xi$ (complete synchronization is replaced by phase synchronization). In the whole interval of $\xi$ pendulum 1 transfers enough energy to pendulum 2 to ensure the pendula's synchronization. The difference of the pendula's displacements $\varphi_{2}-\varphi_{1}$ is so small that it is not visible (in the scale of Figure $3(\mathrm{c})$ ). The case of $k_{\mathrm{x}}=3700[\mathrm{~N} / \mathrm{m}]$ and $m_{\mathrm{b}}=18[\mathrm{~kg}]$ is illustrated in Figure $3(\mathrm{~d})$. In the interval $0.0<\xi<0.49$ one observes periodic rotations of the synchronized pendula (with the phase shift larger than zero). For larger values of $\xi(0.49<\xi<0.70)$ pendula perform periodic synchronous rotations with higher periods or quasiperiodic synchronous rotations. Further increase of $\xi<0.70$ stops pendulum 2 .

In the state of complete synchronization the forces with which pendula act on the beam are algebraically added ${ }^{9,16-18}$ so this example can be generalized to the case of any number of pendula of total mass equal to $m_{1}+m_{2}$. Consider the case of $n$ pendula in the state of complete synchronization. The effect of the switch off of the excitation of $p$ pendula is the same as the effect of switch off of the excitation of pendulum with mass $\left(m_{1}+m_{2}\right) p / n$ in the system of two pendula (the second one with mass $\left.\left(m_{1}+m_{2}\right)(n-p) / n\right)$. As an example consider the system of 20 identical pendula rotating in the same direction with masses $\left(m_{1-20}=0.1[\mathrm{~kg}], m_{\mathrm{b}}=12.0[\mathrm{~kg}], k_{\mathrm{x}}=\right.$ $7000[\mathrm{~N} / \mathrm{m}]$ shown in Figure 3(e-f). All pendula are in the state of complete synchronization when at time $50 \mathrm{~N}$ the excitation of eleven (Figure 3(e)) and twelve (Figure 3(f)) pendula is switched off. Up to the case of 11 pendula the initial complete synchronization is replaced by the phase synchronization and all pendula rotate. The phase shift between the clusters of excited and unexcited pendula 
increases with the increase of the number of unexcited pendula. When the excitation of the 12 pendula is switched off the synchronization is lost and all unexcited pendula stop to rotate as shown in Figure 3(f).

In the considered examples a number of pendula losses excitation simultaneously, if the pendula's excitation is switched off one by one scenario can be different. In the case described in Figure 3(g,h) $\left(m_{1-20}\right.$ $\left.=0.1[\mathrm{~kg}], m_{\mathrm{b}}=12.0[\mathrm{~kg}], k_{\mathrm{x}}=7000[\mathrm{~N} / \mathrm{m}]\right)$ the excitation is switched off at the moments indicated by arrows. In Figure 3(g) eleven pendula are losing excitations in the time intervals of $10 \mathrm{~N}$ starting at $50 \mathrm{~N}$. The increase of the phase shift between the clusters of excited and unexcited pendula is visible. For $N=300$ the excitation of the 12-th pendulum is switched off leading to the loss of synchronization (12 pendula stop to rotate). Different scenario is described in Figure 3(h). The 12-th pendulum loses excitation just after 11-th at $N=160$. Shortly after it 11 pendula (which lost excitation before) stop to rotate but the 12-th pendulum still rotates and is phase synchronized with the cluster of 8 excited pendula (the phase shift is close to $\pi / 4$ ). Later at $N=220$ the 13-th pendulum loses excitation and two clusters of 7 excited and 2 unexcited are created.

Our examples show that it is possible to estimate the critical number of pendula which excitation can be switched off and the rotation of all of them is preserved. In the case when the pendula's excitations are switched off non-simultaneously it is possible to observe the case in which unexcited pendula form two groups one of them stops to rotate and the second one rotates and is phase synchronized with the excited pendula.

The described phenomenon has been observed experimentally Figure 4(a,b). A simple rig consisting of three direct-current electrical motors mounted on the wooden plate which can oscillate horizontally (details on the experimental rig are provided in the Supplementary Information) has been considered. The pendula are mounted at the end of the motor's rods. The control system has been used to vary the angular velocity of pendula's rotation. Figure 4(a) shows the case when all pendula are excited and reach the state of complete synchronization. Next the excitation of the left pendulum has been switched off. Both pendula still rotate (slower than in the previous case) and there is a phase shift between excited pendula and unexcited pendulum as - Figure 4(b).

Thus we have shown that the initial synchronization extends the life-time of the desired behavior of the coupled system. It is possible to estimate the critical number of units which excitation can be switched off and the behavior of the system is not changed qualitatively. The appearance of the phase shift between some units, i.e., transition from the complete to the phase synchronization can be considered as the indicator of the breakdown of excitation in some units. We expect the physical mechanisms that we uncovered here will have important and far-reaching ramifications in the design and use of engineering devices base on coupled systems and in understanding of coupled systems' behavior in nature.

Methods are available in the Supplementary Information.
1. Winfree, A. T. The Geometry of Biological Time, (Springer, New York, 1980).

2. Strogatz, S. H. Sync: The Emerging Science of Spontaneous Order, (Penguin Science, London, 2004).

3. Buck, J. \& Buck, E. Mechanism of synchronous flashing of fireflies. Science 159, 1319-1327 (1968).

4. Neda, Z., Ravasz, E., Brechet, Y., Vicsek, T. \& Barabasi, A.-L. The sound of many hands clapping. Nature 403, 849-850 (2000).

5. Eckhardt, B., Ott, E., Strogatz, S. H., Abrams, D. \& McRobie, A. Modeling walker synchronization on the Millennium Bridge. Phys. Rev. E 75, 021110 (2007).

6. Strogatz, S. H., Abrams, D. M., McRobie, A., Eckhardt, B. \& Ott, E. Crowd synchrony on the Millennium Bridge. Nature 438, 43-44 (2005).

7. Dermott, S. F. How Mercury got its spin. Nature 429, 814-815 (2004).

8. Huygens, C. [Letter to de Sluse]. Oeuveres Completes de Christian Huygens (letters; no. 133 of 24 February 1665, no. 1335 of 26 February 1665, no. 1345 of 6 March 1665), (Societe Hollandaise DesSciences, Martinus Nijhor, La Haye, 1665).

9. Kapitaniak, M., Czolczynski, K., Perlikowski, P., Stefanski, A. \& Kapitaniak, T. Synchronization of clocks. Phys. Rep. 517, 1-67 (2012).

10. Shim, S.-B. Imboden, M. \& Mohanty, P. Synchronized oscillation in coupled nanomechanical oscillators. Science 316, 95-99 (2007).

11. Mancoff, F. B., Rizzo, N. D., Engel, B. N. \& Tehrani, S. Phase-locking in doublepoint-contact spin-transfer devices. Nature 437, 393-395 (2005).

12. Kaka, S. et al. Mutual phase-locking of microwave spin torque nano-oscillators. Nature 437, 389-392 (2005).

13. VanWiggeren, G. D. \& Roy, R. Communication with Chaotic Lasers. Science 279, 1198-1200 (1998).

14. Argyris, A. et al. Chaos-based communications at high bit rates using commercial fibre-optic links. Nature 438, 343-346 (2005).

15. Pikovsky, A., Rosenblum, M. \& Kurths, J. Synchronization: An Universal Concept in Nonlinear Sciences, (Cambridge University Press, Cambridge, 2001).

16. Blekhman, I. I. Synchronization in Science and Technology, (ASME Press, New York, 1988).

17. Czolczynski, K., Perlikowski, P., Stefanski, A. \& Kapitaniak, T. Synchronization of slowly rotating pendulums. Int. J. Bifurcation Chaos Appl. Sci. Eng. 22, 1250128 (2012).

18. Czolczynski, K., Perlikowski, P., Stefanski, A. \& Kapitaniak, T. Synchronization of pendula rotating in different directions. Commun. Nonlinear Sci. Numer. Simul. 17, 3658-3672 (2011).

\section{Acknowledgments}

This work has been supported by the Foundation for Polish Science, Team Programme -Project No TEAM/2010/5/5.

\section{Author contributions}

M.K. and T.K. initiated this work, K.C. performed the modeling and simulations, P.P., M.L. and M.N. build experimental set up and performed experiments. M.K., M.L., M.N., P.P., K.C. and T.K. wrote the paper.

\section{Additional information}

Supplementary information accompanies this paper at http://www.nature.com/ scientificreports

Competing financial interests: The authors declare no competing financial interests.

How to cite this article: Kapitaniak, M., Lazarek, M., Nielaczny, M., Czolczynski, K., Perlikowski, P. \& Kapitaniak, T. Synchronization extends the life time of the desired behavior of globally coupled systems. Sci. Rep. 4, 4391; DOI:10.1038/srep04391 (2014).

(c) This work is licensed under a Creative Commons Attributioncc. visit http://creativecommons.org/licenses/by-nc-sa/3.0 